\title{
Improved Approximation Bounds for Planar Point Pattern Matching ${ }^{\star}$
}

\author{
Minkyoung Cho and David M. Mount \\ Department of Computer Science \\ Institute for Advanced Computer Studies, \\ University of Maryland, College Park MD 20742, USA \\ \{minkcho, mount\}@cs. umd.edu
}

\begin{abstract}
We consider the well known problem of matching two planar point sets under rigid transformations so as to minimize the directed Hausdorff distance. This is a well studied problem in computational geometry. Goodrich, Mitchell, and Orletsky [GMO94] presented a very simple approximation algorithm for this problem, which computes transformations based on aligning pairs of points. They showed that their algorithm achieves an approximation ratio of 4 . We consider a minor modification to their algorithm, which is based on aligning midpoints rather than endpoints. We show that this algorithm achieves an approximation ratio not greater than 3.13. Our analysis is sensitive to the ratio between the diameter of the pattern set and the Hausdorff distance, and we show that the approximation ratio approaches 3 in the limit. Finally, we provide lower bounds that show that our approximation bounds are nearly tight.
\end{abstract}

\section{Introduction}

Geometric pattern matching problem is a fundamental computational problem and has numerous applications in areas such as computer vision [MNM99], image or video compression [ASG02], model-based object recognition [HHW92], and computational chemistry $\left[\mathrm{FKL}^{+} 97\right]$. In general, we are given two point sets, a pattern set $P$ and background set $Q$ from some geometric space. The goal is to compute the transformation $E$ from some geometric group that minimizes some distance measure from $E P$ to $Q$. Throughout, we will consider point sets in the Euclidean plane under rigid transformations (translation and rotation). Distances between two point sets $P$ and $Q$ will be measured by the directed Hausdorff distance, denoted $h(P, Q)$, which is defined to be

$$
h(P, Q)=\max _{p \in P} \min _{q \in Q}\|p q\|,
$$

where $\|p q\|$ denotes the Euclidean distance between points $p$ and $q$. Throughout, unless otherwise specified, we use the term Hausdorff distance to denote

\footnotetext{
* This work was supported by the National Science Foundation under grant CCR0098151.
} 
the directed Hausdorff distance. Thus, our objective is to compute the rigid transformation $E$ that minimizes $h(E P, Q)$.

The problem of point pattern matching has been widely studied. We will focus on methods from the field of computational geometry. Excellent surveys can be found in [AG96] and [Vel01]. There are many variants of this problem, depending on the nature of the inputs, the allowable group of aligning transformations, and the distance function employed. A number of approaches have been proposed for matching points under the Hausdorff distance [AAR94, $\mathrm{CGH}^{+}$97,HKS93]. The computational complexity can be quite high. For example, the best-known algorithm for determining the translation and rotation that minimize the directed Hausdorff distance between sets $P$ and $Q$ of sizes $m$ and $n$, respectively, runs in $O\left(m^{3} n^{2} \log ^{2} m n\right)$ time $\left[\mathrm{CGH}^{+} 97\right]$.

Because of the high complexity of exact point pattern matching, approximation algorithms have been considered. Heffernan and Schirra [HS94] proposed an approximate decision algorithm that tests for congruence within a user-supplied absolute error $\epsilon$. Their algorithm might decline to give a response if the actual distance is too close to $\epsilon$. Alt, Aichholzer and Rote [AAR94] presented a 1.43-factor approximation algorithm for planar point pattern matching under similarity transformations under the bidirectional Hausdorff distance (which is defined to be $\max (h(P, Q), h(Q, P)))$. Neither of these is applicable to our problem, since the cost functions require that every point of $Q$ to have a close match in $P$.

The starting point of this work is the alignment-based approximation algorithms given by Goodrich, Mitchell, and Orletsky [GMO94]. They presented a very simple approximation algorithm for a number of pattern matching formulations, including ours. This is arguably the simplest and most easily implemented algorithm for approximate pattern matching. It operates by computing the transformation that aligns a diametrical pair of points of $P$ with all possible pairs of $Q$, and then returning the transformation with the minimum Hausdorff distance. (We will present the algorithm below.) It runs in $O\left(n^{2} m \log n\right)$ time, and they prove that it achieves an approximation ratio of 4 , that is, it returns a transformation whose Hausdorff distance is at most a factor 4 larger than the optimal Hausdorff distance.

Another closely related piece of work is by Indyk, Motwani, and Venkatasubramanian [IMV99]. They considered the error model of Heffernan and Schirra. They presented an approximation algorithm whose running time is sensitive to the spread $\Delta$ of the point set, which is defined to be the ratio of the distances between the farthest and closest pairs of points. They showed that, for any fixed $\beta>0$, an $(1+\beta)$-approximation can be computed in time $O\left(n^{4 / 3} \Delta^{1 / 3} \log n\right)$. Cardoze and Schulman [CS98] presented a randomized approximation algorithm based on computing convolutions, whose running time is $O\left(n^{2} \log n+\log ^{O(1)} \Delta\right)$ for any fixed precision parameter and any fixed success probability. Although these approaches are asymptotically superior to that of Goodrich et al., they lack its simplicity and ease of implementation. 
In this paper, we reconsider the issue of the approximation ratio for the alignment-based algorithm of Goodrich, Mitchell, and Orletsky. We show that it is possible to improve on the approximation ratio of 4 without altering the simplicity of the algorithm. Our approach is identical in spirit and running time to theirs, but is based on a minor modification of the way in which the aligning transformation is computed. We call our modification symmetric alignment, because it is based on aligning midpoints, rather than endpoints. We show that the resulting approximation ratio is never more than 3.13 (a quantity resulting from the numerical solution of an equation).

We also analyze the approximation ratio as a function of a parameter that depends on the closeness of the optimal match. Let $\rho$ be half the ratio of the diameter of $P$ to the minimum Hausdorff distance. We show that our algorithm achieves an approximation ratio of at most $3+\frac{1}{\sqrt{3} \rho}$. In many applications $\rho$ can be quite large. Examples include document analysis and satellite image analysis, where the ratio of diameter of the pattern ranges from tens to hundreds of pixels, while the expected digitization error is roughly one pixel. For these applications, the approximation ratio will be quite close to 3 . We also show that for sufficiently large $\rho$, the approximation factor is at least $3+\frac{1}{10 \rho^{2}}$.

It is worth noting that the more sophisticated approximation algorithm of Indyk, et al. [IMV99] uses the simple alignment algorithm as a subroutine. The running time of their algorithm has a cubic dependence on the approximation ratio of the alignment algorithm. So, an improvement in the approximation ratio has the effect of reducing the running time of their algorithm as well.

The remainder of the paper is organized as follows. In Section 2 we present the algorithm of [GMO94], which we call the serial alignment algorithm and review the derivation of its approximation bounds. Next, we will introduce the symmetric alignment algorithm, and derive a crude analysis of its approximation ratio. In Section 3, we will present a more accurate analysis of the approximation bound. Finally, in Section 4 we give concluding remarks.

\section{The Serial and Symmetric Alignment Algorithms}

In this section we present a description of the serial alignment algorithm of Goodrich, Mitchell, and Orletsky [GMO94] and review its approximation bound. We also described our modification of this algorithm, called symmetrical alignment. Recall that $P=\left\{p_{1}, \ldots, p_{m}\right\}$ is the pattern set and $Q=\left\{q_{1}, \ldots, q_{n}\right\}$ is the background set to search, and we seek a rigid transformation of $P$ that minimizes the Hausdorff distance with $Q$.

The serial alignment algorithm proceeds as follows. Let $\left(p_{1}, p_{2}\right)$ denote a pair of points having the greatest distance in $P$, that is, a diametrical pair. For each distinct pair $\left(q_{1}, q_{2}\right)$ in $Q$, the algorithm computes a rigid transformation matching $\left(p_{1}, p_{2}\right)$ with $\left(q_{1}, q_{2}\right)$ as follows. First, it determines a translation that maps $p_{1}$ to $q_{1}$ and then a rotation about $p_{1}$ that aligns the directed line segment $\overrightarrow{p_{1} p_{2}}$ with $\overrightarrow{q_{1} q_{2}}$. The composition of these two transformations is then applied to the entire pattern set $P$ and the Hausdorff distance is computed. At the end, the algorithm returns the rigid transformation that achieves the minimum Hausdorff 
distance. The running time of this algorithm is $O\left(n^{2} m \log n\right)$ because, for each of the $n(n-1)$ distinct pairs of $Q$, we compute the aligning transformation $E$ in $O(1)$ time, and then, for each point $p \in P$, we compute the distance from $E(p)$ to its nearest neighbor in $Q$. Nearest neighbors queries in a planar set $Q$ can be answered in time $O(\log n)$ after $O(n \log n)$ preprocessing [dBvKOS00]. Goodrich, Mitchell, and Orletsky prove that this algorithm achieves an approximation ratio of 4 . For completeness let us summarize their argument.

Lemma 1. (Goodrich, Mitchell, and Orletsky [GMO94]) The serial alignment algorithm has an approximation ratio of at most 4 .

Proof. For simplicity of notation, let us assume that $P$ has been presented to the algorithm in its optimal position with respect to $Q$. Let $h_{\text {opt }}$ denote the optimal Hausdorff distance from $P$ to $Q$. This means that for each point of $P$ there exists a point of $Q$ within distance $h_{\text {opt }}$. Now, we run the above algorithm and bound the maximum distance by which any point of $P$ is displaced relative to its original (optimal) position. This maximum displacement distance will give an approximation bound relative to the optimal distance $h_{\text {opt }}$.

Clearly, in the process of translation, each point of the pattern set $P$ moves by at most $h_{\text {opt }}$. To determine the displacement due to rotation, it suffices to consider $p_{2}$ since it is the farthest point from $p_{1}$. The point $p_{2}$ was initially within distance $h_{\text {opt }}$ of its corresponding points of $Q$, denoted $q_{2}$, and the above translation moves it by an additional distance of at most $h_{\text {opt }}$. Thus, rotating $p_{2}$ into alignment with $q_{2}$ moves it by a distance of at most $2 h_{\text {opt }}$. Therefore, given that it started within distance $h_{\text {opt }}$ of some point of $Q$, it follows that its contribution to the Hausdorff distance is at most $h_{\text {opt }}+2 h_{\text {opt }}+h_{\text {opt }}=4 h_{\text {opt }}$, which establishes the approximation bound.

There are two obvious shortcomings with this algorithm and its analysis. The first is that the algorithm does not optimally align the pair $\left(p_{1}, p_{2}\right)$ with the pair $\left(q_{1}, q_{2}\right)$ with respect to Hausdorff distance. One would expect such an optimal alignment to provide a better approximation bound. This observation is the basis of our algorithm. The second shortcoming is that the analysis fails to account for the fact that the displacement distances due to translation and rotation are functions of the points' relative position to $p_{1}$ and $p_{2}$, and so share some dependency.

Next, we describe our approach, called symmetrical alignment. The algorithm differs only in how the aligning transformation is computed, given the pairs $\left(p_{1}, p_{2}\right)$ in $P$ and $\left(q_{1}, q_{2}\right)$ in $Q$. Let $m_{p}$ and $m_{q}$ denote the respective midpoints of line segments $\overline{p_{1} p_{2}}$ and $\overline{q_{1} q_{2}}$. First, translate $P$ to map $m_{p}$ to $m_{q}$ and then rotate $P$ about $m_{p}$ to align the directed segments $\overrightarrow{p_{1} p_{2}}$ with $\overrightarrow{q_{1} q_{2}}$. Thus, the only difference is that we align and rotate around the midpoints rather than $p_{1}$. Observe that this alignment transformation minimizes the Hausdorff distance between the pairs $\left(p_{1}, p_{2}\right)$ and $\left(q_{1}, q_{2}\right)$.

Before giving our detailed analysis of the approximation bound, we establish a crude approximation bound, which justifies the benefit of symmetric alignment. 
Lemma 2. The symmetric alignment algorithm has an approximation ratio of at most $(2+\sqrt{3}) \approx 3.732$.

Proof. We will apply the same approach used in the analysis of serial alignment. As before, let us assume that $P$ has been presented to the algorithm in its optimal position with respect to $Q$. (Note that the algorithm's final alignment is independent of the initial point placement.) Let $\boldsymbol{v}_{1}$ and $\boldsymbol{v}_{2}$ denote the vector forms of $\overrightarrow{p_{1} q_{1}}$ and $\overrightarrow{p_{2} q_{2}}$, respectively. To match the midpoints $m_{p}$ and $m_{q}$, we translate the pattern set $P$ by $\left(\boldsymbol{v}_{1}+\boldsymbol{v}_{2}\right) / 2$. Let $T$ denote this translation. The distance between $T\left(p_{1}\right)$ and $q_{1}$ is $\left\|\boldsymbol{v}_{1}-\left(\boldsymbol{v}_{1}+\boldsymbol{v}_{2}\right) / 2\right\|=\left\|\left(\boldsymbol{v}_{1}-\boldsymbol{v}_{2}\right) / 2\right\| \leq h_{\text {opt }}$. By symmetry, the same bound applies to the distance from $T\left(p_{2}\right)$ to $q_{2}$. Therefore, $T\left(p_{1}\right)$ will move by at most $h_{\text {opt }}$ during the rotation process.

Because $\left(p_{1}, p_{2}\right)$ is a diametrical pair, it follows that all the points of $P$ lie in a lune defined by the intersection of two discs, both of radius $\left\|p_{1} p_{2}\right\|$, centered at these points. Thus, no point of $P$ is farther from their midpoint than the apex of the lune, which is at distance $(\sqrt{3} / 2)\left\|p_{1} p_{2}\right\|$. Since this rotation moves $p_{1}$ (or equivalently $p_{2}$ ) by a distance of at most $h_{\text {opt }}$, and $p_{1}$ is within distance $(1 / 2)\left\|p_{1} p_{2}\right\|$ of $m_{p}$, it follows that any point $p \in P$ is moved by at most $\sqrt{3} h_{\text {opt }}$. Given that $p$ started within distance $h_{\text {opt }}$ of some point of $Q$, its final contribution to the Hausdorff distance is at most $h_{\text {opt }}+\sqrt{3} h_{\text {opt }}+h_{\text {opt }}=$ $(2+\sqrt{3}) h_{\text {opt }} \approx 3.732 h_{\text {opt }}$.

Even though this crude approximation bound is already an improvement, it suffers from the same problem as the earlier analysis in that it does not consider the geometric relationship between the translation and rotation vectors

\section{Main Results}

In this section, we will prove a more exact approximation bound for symmetric alignment. As before, let $h_{\text {opt }}$ denote the optimal Hausdorff distance between $P$ and $Q$ achievable under any rigid transformation of $P$. Let $A_{\text {sym }}(\rho)$ denote the approximate ratio for symmetric alignment.

Theorem 1. Consider two planar point sets $P$ and $Q$ whose optimal Hausdorff distance under rigid transformations is $h_{\text {opt }}$. Let $\rho=\frac{1}{2} \operatorname{diam}(P) / h_{\text {opt }}$, where $\operatorname{diam}(P)$ denotes the diameter of $P$. Then the for all $\rho>0$, the approximation ratio of symmetric alignment satisfies:

$$
A_{\text {sym }}(\rho) \leq \min \left(3+\frac{1}{\sqrt{3} \rho}, \sqrt{4 \rho^{2}+2 \rho+1}\right) .
$$

In typical applications where $\rho$ is relatively large, this shows that the approximation bound is nearly 3 . The remainder of this section is devoted to proving this theorem. As usual, it will simplify notation to assume that $P$ has been transformed to its optimal placement with respect to $Q$, and we will bound the amount of additional displacement of each point of $P$ relative to this placement. Without loss of generality we may scale space uniformly so that $h_{\text {opt }}=1$, implying that for each point of $P$ there exists at least one point of $Q$ within distance 1 . 
The algorithm starts by computing a pair $\left(p_{1}, p_{2}\right)$ of points of $P$ with the greatest distance. Let $\left(q_{1}, q_{2}\right)$ denote the corresponding pair of points of $Q$. From our scaling it follows that for $i \in\{1,2\}, q_{i}$ lies within a unit disc centered at $p_{i}$. (See Fig. 1.) Let $m_{p}$ and $m_{q}$ denote the respective midpoints of the segments $\overline{p_{1} p_{2}}$ and $\overline{q_{1} q_{2}}$. Note that $\rho$ is just the distance from $m_{p}$ to either $p_{1}$ or $p_{2}$. Let $\alpha$ denote the angle that aligns the directed segments $\overrightarrow{p_{1} q_{1}}$ with $\overrightarrow{p_{2} q_{2}}$. Without loss of generality, we may assume this angle is acute. If $\rho>1$, then the two unit discs do not intersect, and it follows easily that $0 \leq \sin \alpha \leq 1 / \rho$.

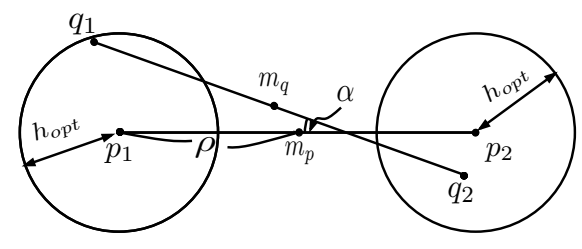

Fig. 1. The positions of the point sets prior to running the algorithm.

Here is a brief overview of the proof. We will establish two approximation bounds, each a function of $\rho$. One bound is better for low values of $\rho$ and the other is better for high values. The crossover value of these functions is denoted $\rho^{*}$, and we will show that its value is approximately 1.26. Due to space limitations, we only present the approximation bound for the more interesting case, when $\rho>\rho^{*}$.

We begin by considering the space of possible translations that align $m_{p}$ with $m_{q}$. It will make matters a bit simpler to think of translating $Q$ to align $m_{q}$ with $m_{p}$, but of course any bounds on the distance of translation will apply to case of translating $P$. The following lemma bounds the translation distance, which we will denote by $\|T\|$, as a function of $\rho$ and $\alpha$. Note that the $\alpha$ is not affected by the translation.
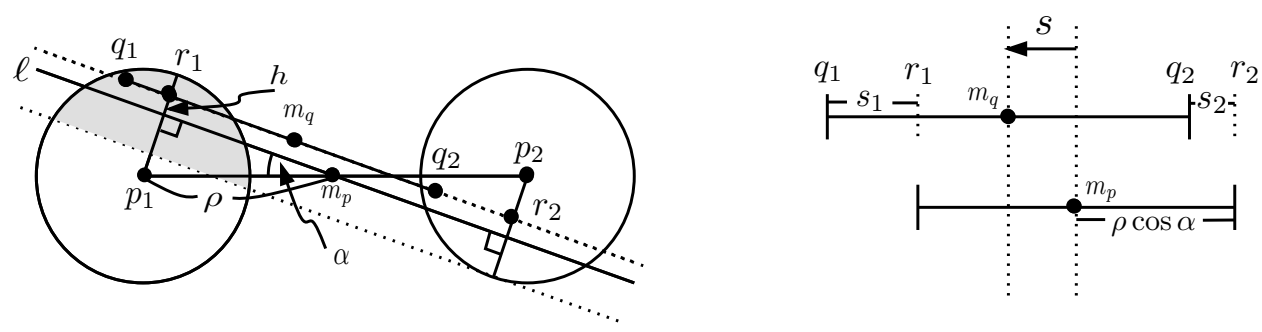

Fig. 2. The analysis of the midpoint translation.

Lemma 3. The symmetric alignment's translation transformation displaces any point of $P$ by a vector $T \in \mathcal{T}_{\rho}(\alpha)$ satisfying $\|T\| \leq \sqrt{1-\rho^{2} \sin ^{2} \alpha}$. 
Proof. Consider a line passing through $m_{p}$ that is parallel to $\overline{q_{1} q_{2}}$. Let $r_{1}$ and $r_{2}$ be the respective orthogonal projections of $p_{1}$ and $p_{2}$ onto the line $\overline{q_{1} q_{2}}$ and let $s_{1}$ and $s_{2}$ denote the signed distances $\left\|\overrightarrow{r_{1} q_{1}}\right\|$ and $\left\|\overrightarrow{r_{2} q_{2}}\right\|$, respectively. Consider a coordinate system centered at $m_{p}$ whose positive $s$-axis is located along a line $\ell$ that is directed in the sense of $\overrightarrow{q_{2} q_{1}}$, and whose positive $h$-axis is perpendicular to this and directed away from $\ell$ towards the line $\overline{q_{1} q_{2}}$. In this coordinate system we have $m_{p}=(0,0), q_{1}=\left(s_{1}+\rho \cos \alpha, h\right)$, and $q_{2}=\left(s_{2}-\rho \cos \alpha, h\right)$. Thus, $m_{q}=$ $\left(\left(s_{1}+s_{2}\right) / 2, h\right)$, and the translation vector $T$ is $m_{q}-m_{p}=m_{q}$. By straightforward calculations we have $\left|s_{1}\right| \leq \sqrt{1-(\rho \sin \alpha+h)^{2}}$ and $\left|s_{2}\right| \leq \sqrt{\left(1-(\rho \sin \alpha+h)^{2}\right.}$. Therefore,

$$
\begin{aligned}
\|T\|^{2} & =s^{2}+h^{2}=\left(\frac{s_{1}+s_{2}}{2}\right)^{2}+h^{2}=\frac{1}{2}\left(s_{1}^{2}+s_{2}^{2}\right)-\frac{1}{4}\left(s_{1}-s_{2}\right)^{2}+h^{2} \\
& \leq \frac{1}{2}\left(s_{1}^{2}+s_{2}^{2}\right)+h^{2} \leq \frac{1}{2}\left\{\left(1-(\rho \sin \alpha+h)^{2}\right)+\left(1-(\rho \sin \alpha-h)^{2}\right)\right\}+h^{2} \\
& =1-\rho^{2} \sin ^{2} \alpha .
\end{aligned}
$$

Observe that the translation is maximized when $s_{1}=s_{2}$, and this means that the line $\overline{q_{1} q_{2}}$ passes through $m_{p}$.

Next we consider the effect of rotation on the approximation error. Unlike translation we need to consider the placement of points in $P$ because the distance by which a point is moved by rotation is determined by both the rotation angle $\alpha$ and the distance from this point to the center of rotation. As mentioned in the proof of Lemma 2 every point of $P$ lies within a lune formed by the intersection of two discs of radius $2 \rho$ centered at $p_{1}$ and $p_{2}$. (See Fig. 3.) The following lemma describes the possible displacements of a point of $P$ under the rotational part of the aligning transformation. This is done relative to a coordinate system centered at $m_{p}$, whose $x$-axis is directed towards $p_{2}$.

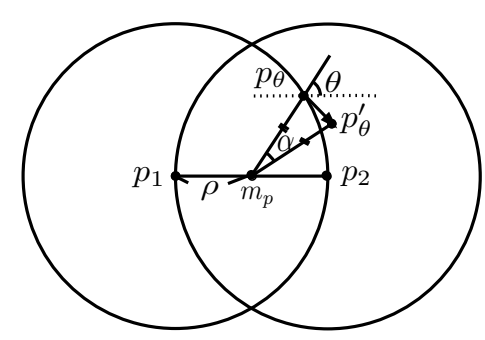

Fig. 3. The proof of rotation.

Lemma 4. The symmetric alignment's rotation transformation displaces any point of $P$ by a vector $R$ satisfying $\|R\| \leq 2 \sqrt{3} \rho \sin \frac{\alpha}{2}$. 
Proof. Let $p_{\theta}$ be any point of $P$ such that the signed angle from $\overrightarrow{m_{p} p_{2}}$ to $\overrightarrow{m_{p} p_{\theta}}$ is $\theta$. Let $p_{\theta}^{\prime}$ denote the point after rotating $p_{\theta}$ counterclockwise about $m_{p}$ by angle $\alpha$. By simple trigonometry and the observation that $\triangle m_{p} p_{\theta} p_{\theta}^{\prime}$ is isosceles, it follows that the length of the displacement $\overrightarrow{p_{\theta} p_{\theta}^{\prime}}$, which we denote by $\|R\|$, is $\left\|m_{p} p_{\theta}\right\| \cdot 2 \sin \frac{\alpha}{2}$. The farthest point of the lune from $m_{p}$ is easily seen to be the apex, which is at distance $\sqrt{3} \rho$. Thus, $\|R\|$ is at most $2 \sqrt{3} \rho \sin \frac{\alpha}{2}$.

We are now ready to derive the approximation bound on the symmetric alignment algorithm by combining the bounds on the translational and rotational displacements. Recall that at the start of the algorithm, the points are assumed to placed in the optimal positions and that space has been scaled so that $h_{\text {opt }}=1$. It follows that each point of $P$ has been displaced from its initial position within unit distance of a point of $Q$ to a new position that is now within distance $\left\|T_{\rho}(\alpha)+R_{\rho}(\alpha)\right\|+1$ of some point of $Q$. For any fixed value of $\rho$ it follows that the approximation bound $A_{\text {sym }}(\rho)$ is at most

$$
A_{\text {sym }}(\rho)=\max _{\alpha}\left\|T_{\rho}(\alpha)+R_{\rho}(\alpha)\right\|+1 .
$$

Recall that $0 \leq \sin \alpha \leq 1 / \rho$.

Unfortunately, determining this length bound exactly would involve solving a relatively high order equation involving trigonometric functions. Instead, we will compute an upper bound by applying the triangle inequality to separate the translation and rotation components.

$$
\begin{aligned}
A_{\text {sym }}(\rho) & \leq \max _{\alpha}\left(\left\|T_{\rho}(\alpha)\right\|+\left\|R_{\rho}(\alpha)\right\|\right)+1 \\
& \left.=\max _{\alpha}\left(\sqrt{1-\rho^{2} \sin ^{2} \alpha}+2 \sqrt{3} \rho \sin \frac{\alpha}{2}\right)+1 \quad \text { (from Lemmas } 3 \text { and } 4\right) .
\end{aligned}
$$

Substituting $x=\cos \alpha$, it follows that the quantity to be maximized is

$$
f_{\rho}(x)=\sqrt{1-\rho^{2}\left(1-x^{2}\right)}+\sqrt{6} \rho \sqrt{1-x}+1, \quad \text { where } \sqrt{1-\frac{1}{\rho^{2}}} \leq x \leq 1 .
$$

To find the maximum of $f_{\rho}$, we take the partial derivative with respect to $x$.

$$
\frac{\partial f_{\rho}}{\partial x}=-\frac{\sqrt{3} \rho}{\sqrt{2} \sqrt{1-x}}+\frac{\rho^{2} x}{\sqrt{1-\rho^{2}\left(1-x^{2}\right)}} .
$$

By our earlier assumption that $\rho \geq \rho^{*} \approx 1.26$, it follows by symbolic manipulations that $\partial f_{\rho} / \partial x=0$ has a single real root for $\rho \geq \rho^{*}$, which is

$$
\begin{aligned}
x_{0}= & \frac{1}{6}\left(-1+c_{1}(\rho)+\frac{1}{c_{1}(\rho)}\right), \\
& \text { where } \quad c_{1}(\rho)=\frac{\rho^{2}}{\left(161 \rho^{6}+18 \rho^{4} \sqrt{c_{2}(\rho)}-162 \rho^{4}\right)^{1 / 3}} \\
& \text { and } \quad c_{2}(\rho)=80 \rho^{4}-161 \rho^{2}+81 .
\end{aligned}
$$


By an analysis of this derivative it follows that the function $f_{\rho}$ achieves its maximum value when $x=x_{0}$, and so the final approximation bound is $f_{\rho}\left(x_{0}\right)=$ $\sqrt{1-\rho^{2}\left(1-x_{0}^{2}\right)}+\sqrt{6} \rho \sqrt{1-x_{0}}+1$. By computing the partial derivation of $f_{\rho}(x)$ with respect to $\rho$ that, for any fixed $x$, this function is a monotonically decreasing function of $\rho$.

Unfortunately, this function is too complex to reason about easily. Nonetheless, we can evaluate it for any fixed value of $\rho$. The resulting approximation bound is plotted as a function of $\rho$ in Fig. 4 below. The figure shows that as $\rho$ increases, the approximation bound converges to 3 . The following result establishes this asymptotic convergence by proving a somewhat weaker approximation bound.

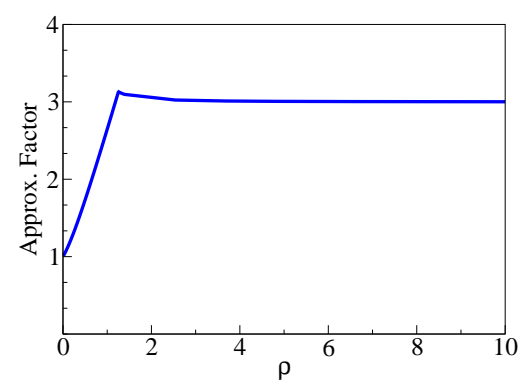

Fig. 4. The approximation bound for symmetric alignment as a function of $\rho$.

Lemma 5. If $\rho>1.5$ then the approximation bound of the symmetric alignment algorithm, $A_{\text {sym }}(\rho)$, is at most $3+\frac{1}{\sqrt{3} \rho}$.

Proof. Before presenting the general case, we consider the simpler limiting case when $\rho$ tends to $\infty$. Since $0 \leq \sin \alpha \leq 1 / \rho$, in the limit $\alpha$ approaches 0 . Using the fact of $\lim _{\alpha \rightarrow 0} \frac{\sin \alpha}{\alpha}=1$ we have

$$
\begin{aligned}
A_{\text {sym }}(\infty) & =\lim _{\rho \rightarrow \infty} A_{\text {sym }}(\rho)=\lim _{\rho \rightarrow \infty} \sqrt{1-\rho^{2} \sin ^{2} \alpha}+2 \sqrt{3} \rho \sin \frac{\alpha}{2}+1 \\
& =\sqrt{1-\rho^{2} \alpha^{2}}+\sqrt{3} \rho \alpha+1 .
\end{aligned}
$$

Let $x=\rho \alpha$. In the limit we have $0 \leq x \leq 1$ and so

$$
A_{\text {sym }}(\infty) \leq \max _{0 \leq x \leq 1}\left(\sqrt{1-x^{2}}+\sqrt{3} x+1\right) .
$$

It is easy to verify that $A_{\text {sym }}(\infty)$ achieves a maximum value of 3 when $x=\sqrt{3} / 2$.

Next, we consider the general case. Since $\rho>1.5$ and $\sin \alpha \leq 1 / \rho$, it follows that $0 \leq x \leq 1.1$. We consider two cases, $0 \leq x \leq 1$ and $1<x \leq 1.1$. We consider only the first case, due to space limitations. To simplify $A_{\text {sym }}(\rho)$, we 
use a Taylor series expansion and the fact that $A_{\text {sym }}(\infty) \leq 3$.

$$
\begin{aligned}
A_{\text {sym }}(\rho) & \leq \max _{\alpha}\left(\sqrt{1-\rho^{2} \sin ^{2} \alpha}+2 \sqrt{3} \rho \sin \frac{\alpha}{2}+1\right) \\
& \leq \max _{\alpha}\left(\sqrt{1-\rho^{2}\left(\alpha-\alpha^{3} / 3\right)^{2}}+\sqrt{3} \rho \alpha+1\right) \\
& \leq \max _{\alpha}\left(\sqrt{1-\rho^{2}\left(\alpha-\alpha^{3} / 6\right)^{2}}+\sqrt{3} \rho \alpha-\left(\sqrt{1-\rho^{2} \alpha^{2}}+\sqrt{3} \rho \alpha+1\right)+3+1\right) \\
& =\max _{\alpha}\left(\sqrt{1-\rho^{2}\left(\alpha-\alpha^{3} / 6\right)^{2}}-\sqrt{1-\rho^{2} \alpha^{2}}+3\right) .
\end{aligned}
$$

Substituting $x=\rho \alpha$, let

$$
g(x)=\sqrt{1-x^{2}\left(1-\frac{x^{2}}{6 \rho^{2}}\right)^{2}}-\sqrt{1-x^{2}}+3 .
$$

For all fixed $\rho$, this is a monotonically increasing function in $x$. Thus, since $x \leq 1$, this function achieves its maximum value at $x=1$ of

$$
g(1)=3+\sqrt{1-\left(1-\frac{1}{6 \rho^{2}}\right)^{2}}=3+\sqrt{\frac{1}{3 \rho^{2}}-\frac{1}{36 \rho^{4}}} \leq 3+\frac{1}{\sqrt{3} \rho} .
$$

\subsection{A Lower Bound for Symmetric Alignment}

It is natural to wonder whether the upper bounds on the approximation ratio $A_{\text {sym }}$ proved in Theorem 1 are tight. Next, we prove that this approximation bound is close to tight. We show that for all sufficiently large $\rho$ the approximation factor is strictly greater than 3 , and approaches 3 in the limit.

Lemma 6. For all $\rho>2 A_{\text {sym }}$, there exists an input on which symmetric achieves an approximation factor of at least $3+\frac{1}{10 \rho^{2}}$.

The remainder of this section is devoted to proving this. We consider two configurations of points. Consider a fixed value $\rho>2 A_{\text {sym }}$. We define the pattern point set $P=\left\{p_{1}, p_{2}, p_{3}, p_{4}\right\}$, where the first three points form an equilateral triangle of side length $2 \rho$, and place $p_{4}$ at the midpoint of $\overline{p_{1} p_{2}}$. (See Fig. 5.) By an infinitesimal perturbation of the points, we may assume that the pair $\left(p_{1}, p_{2}\right)$ is the unique diametrical pair for $P$.

Next, to define the locations of the other background set $Q=\left\{q_{1}, q_{2}, q_{3}, q_{4}\right\}$, let $C_{i}$ denote a circle of unit radius centered $p_{i}$. Consider a line passing through $p_{4}$ (the midpoint of $\overline{p_{1} p_{2}}$ ) forming an angle $\alpha$ with line $\overline{p_{1} p_{2}}$, where $\sin \alpha=\frac{\sqrt{3}}{2 \rho}$. Place $q_{1}$ and $q_{2}$ at the rightmost intersection points of the line and $C_{1}$ and $C_{2}$, respectively. Let $\boldsymbol{t}$ and $\boldsymbol{r}$ denote the translation and rotation displacement vectors resulting from symmetric alignment, respectively. (They are described 

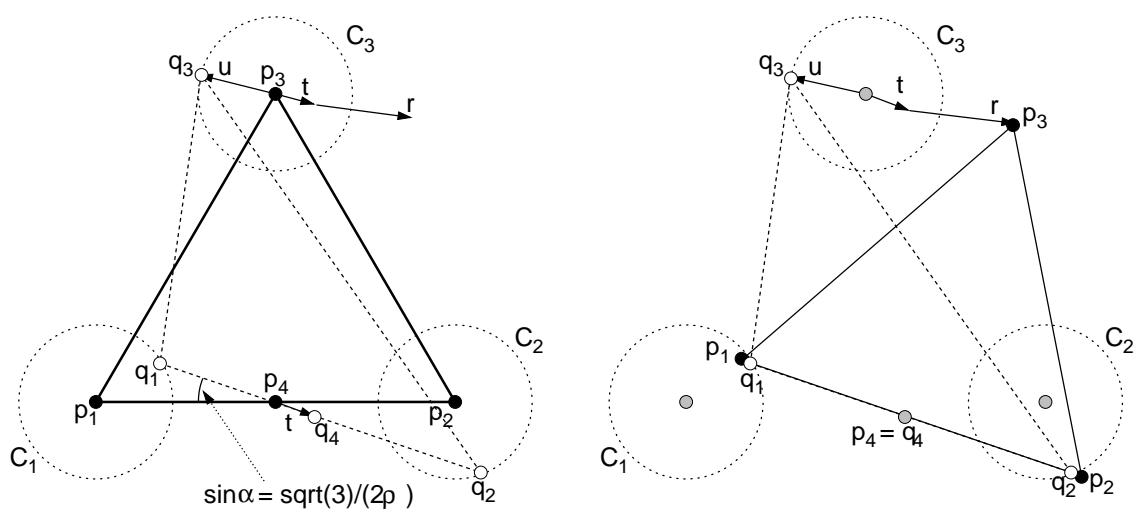

Fig. 5. The lower bound on $A_{\text {sym }}$.

below.) Let $q_{3}=p_{3}+\boldsymbol{u}$, where $\boldsymbol{u}$ is a unit length vector whose direction is chosen to be directly opposite that of $\boldsymbol{t}+\boldsymbol{r}$. Finally, place $q_{4}$ at the midpoint of $\overline{q_{1} q_{2}}$. Observe that for each $p_{i}$, the corresponding point $q_{i}$ lies within distance 1 .

For this placement, the directed Hausdorff distance is exactly 1, and so $h_{\text {opt }} \leq 1$. We run the symmetric alignment algorithm on $P$ and $Q$, and consider the displacement distance of $p_{3}$ relative to $q_{3}$. The fact that $\rho>2 A_{\text {sym }}$ and the presence of $p_{4}$ and $q_{4}$ imply that the final matching chosen by symmetric alignment results by aligning $\left(p_{1}, p_{2}\right)$ with $\left(q_{1}, q_{2}\right)$. The reason is that any other choice will result in $p_{4}$ having no point within distance $A_{\text {sym }}$, thus leading to a contradiction. (Details have been omitted due to space limitations.)

By adapting the analyses of Lemmas 3 and 4, it can be seen that this configuration achieves the worst case for these two lemmas. In particular, the length of the translation displacement is $\|\boldsymbol{t}\|=\sqrt{1-\rho^{2} \sin ^{2} \alpha}=\sqrt{1-(3 / 4)}=1 / 2$, and the length of the rotation displacement is $\|\boldsymbol{r}\|=2 \sqrt{3} \rho \sin \frac{\alpha}{2}$. Furthermore, it is easy to show that the angle between these two vectors is $\frac{\alpha}{2}$.

To complete the analysis, we decompose the rotation displacement vector $\boldsymbol{r}$ into two components, $\boldsymbol{r}_{1}$ is parallel to $\boldsymbol{t}$ and $\boldsymbol{r}_{2}$ is perpendicular to it, and apply the fact that the angle between these vectors is $\alpha / 2$. After some algebraic manipulations, it follows that the squared magnitude of the final displacement is at least $4+\frac{27}{64 \rho^{2}}$, and under the assumption that $\rho>2 A_{\text {sym }} \geq 2$, the bound given in the statement of the lemma follows. Details have been omitted due to space limitations.

\section{Concluding Remarks}

We have presented a simple modification to the alignment-based algorithm of Goodrich, Mitchell, and Orletsky [GMO94]. Our modification has the same running time and retains the simplicity of the original algorithm. We have shown 
that, in contrast to the factor-4 approximation bound proved in [GMO94], our approach has an approximation ratio that is never larger than 3.13, and converges to 3 in the limit as the ratio $\rho$ between the pattern set diameter and the Hausdorff distance increases. We have also shown that the bound is nearly tight. This paper opens the question of what are the performance limits of approximations based on point alignments. It is natural to consider generalizations of this approach to higher dimensions, to matchings based on alignments of more than two points, and to other sorts of distance measures and transformation groups.

\section{References}

[AAR94] H. Alt, O. Aichholzer, and G. Rote. Matching shapes with a reference point. In Proc. 10th Annu. ACM Sympos. Comput. Geom., pages 85-92, 1994.

[AG96] H. Alt and L. J. Guibas. Discrete geometric shapes: Matching, interpolation, and approximation. Technical Report 96-11, Institut für Informatik, Freie Unversität Berlin, Berlin, Germany, 1996.

[ASG02] M. Alzina, W. Szpankowski, and A. Grama. 2d-pattern matching image and video compression: Theory, algorithms, and experiments. IEEE Trans. on Image Processing, 11:318-331, 2002.

$\left[\mathrm{CGH}^{+} 97\right] \quad$ L. P. Chew, M. T. Goodrich, D. P. Huttenlocher, K. Kedem, J. M. Kleinberg, and D. Kravets. Geometric pattern matching under Euclidean motion. Comput. Geom. Theory Appl., 7:113-124, 1997.

[CS98] D. E. Cardoze and L. Schulman. Pattern matching for spatial point sets. In Proc. 39th Annu. IEEE Sympos. Found. Comput. Sci., pages 156-165, 1998.

[dBvKOS00] M. de Berg, M. van Kreveld, M. H. Overmars, and O. Schwarzkopf. Computational Geometry: Algorithms and Applications. Springer-Verlag, 2nd edition, 2000.

$\left[\mathrm{FKL}^{+} 97\right]$ P. Finn, L. E. Kavraki, J. C. Latombe, R. Motwani, C. Shelton, S. Venkatasubramanian, and A. Yao. Rapid: Randomized pharmacophore identification for drug design. In Proc. 13th Annu. ACM Sympos. Comput. Geom., pages 324-333, 1997.

[GMO94] M. T. Goodrich, J. S. Mitchell, and M. W. Orletsky. Practical methods for approximate geometric pattern matching under rigid motion. In Proc. 10th Annu. ACM Sympos. Comput. Geom., pages 103-112, 1994.

[HHW92] J. E. Hopcroft, D. P. Huttenlocher, and P. C. Wayner. Affine invariants for model-based recognition. MIT Press, Cambridge, 1992.

[HKS93] D. P. Huttenlocher, K. Kedem, and M. Sharir. The upper envelope of Voronoi surfaces and its applications. Discrete Comput. Geom., 9:267291, 1993.

[HS94] P. J. Heffernan and S. Schirra. Approximate decision algorithms for point set congruence. Comput. Geom. Theory Appl., 4:137-156, 1994.

[IMV99] P. Indyk, R. Motwani, and S. Venkatasubramanian. Geometric matching under noise: Combinatorial bounds and algorithms. In Proc. 8th Annual ACM-SIAM on Discrete Algorithm, pages 457-465, 1999.

[MNM99] D. M. Mount, N. S. Netanyahu, and J. Le Moigne. Efficient algorithms for robust point pattern matching. Pattern Recognition, 32:17-38, 1999. 
[Vel01] R. C. Veltkamp. Shape matching: Similarity measures and algorithms. Technical Report UU-CS 2001-03, Utrecht University: Information and Computing Sciences, Utrecht, The Netherlands, 2001. 